\title{
The macroevolution of size and complexity in insect male genitalia
}

Andrey Rudoy, Ignacio Ribera

The evolution of insect male genitalia has received much attention, but there is still a lack of data on the macroevolutionary origin of its extraordinary variation. We used a calibrated molecular phylogeny of 71 of the 150 known species of the beetle genus Limnebius to study the evolution of the size and complexity of the male genitalia in its two subgenera, Bilimneus, with small species with simple genitalia, and Limnebius s.str., with a much larger variation in size and complexity. We reconstructed ancestral values of complexity (perimeter and fractal dimension of the aedeagus) and genital and body size with Bayesian methods. Complexity evolved more in agreement with a Brownian model, although with evidence of weak directional selection to a decrease or increase in complexity in the two subgenera respectively, as measured with an excess of branches with negative or positive change. On the contrary, aedeagus size, the variable with the highest rates of evolution, had a lower phylogenetic signal, without significant differences between the two subgenera in the average change of the individual branches of the tree. Aedeagus size also had a lower correlation with time and no evidence of directional selection. Rather than to directional selection, it thus seems that the higher diversity of the male genitalia in Limnebius s.str. is mostly due to the larger variance of the phenotypic change in the individual branches of the tree for all measured variables. 


\section{The macroevolution of size and complexity in insect male genitalia}

2

\section{Andrey Rudoy and Ignacio Ribera}

4

5 Institute of Evolutionary Biology (CSIC-Universitat Pompeu Fabra), Passeig Maritim de la

6 Barceloneta 37-49, 08003 Barcelona, Spain

8

9 Author for correspondence: Ignacio Ribera. e-mail: ignacio.ribera@ibe.upf-csic.es

10

11

12

13

14

15

16 


\section{ABSTRACT}

18 The evolution of insect male genitalia has received much attention, but there is still a lack of data

19 on the macroevolutionary origin of its extraordinary variation. We used a calibrated molecular

20 phylogeny of 71 of the 150 known species of the beetle genus Limnebius to study the evolution

21 of the size and complexity of the male genitalia in its two subgenera, Bilimneus, with small

22 species with simple genitalia, and Limnebius s.str., with a much larger variation in size and

23 complexity. We reconstructed ancestral values of complexity (perimeter and fractal dimension of

24 the aedeagus) and genital and body size with Bayesian methods. Complexity evolved more in 25 agreement with a Brownian model, although with evidence of weak directional selection to a 26 decrease or increase in complexity in the two subgenera respectively, as measured with an excess 27 of branches with negative or positive change. On the contrary, aedeagus size, the variable with 28 the highest rates of evolution, had a lower phylogenetic signal, without significant differences 29 between the two subgenera in the average change of the individual branches of the tree.

30 Aedeagus size also had a lower correlation with time and no evidence of directional selection.

31 Rather than to directional selection, it thus seems that the higher diversity of the male genitalia in

32 Limnebius s.str. is mostly due to the larger variance of the phenotypic change in the individual

33 branches of the tree for all measured variables.

35 Keywords: aedeagus, Coleoptera, directional selection, rates of evolution 


\section{INTRODUCTION}

Insect genitalia have been the focus of much attention since the middle XIX century, when their taxonomic value to diagnose species with otherwise very similar external morphologies was recognised. It soon became apparent that this variability should play a prominent role in reproductive isolation and speciation (e.g. Dufour, 1848), generating much debate on the causes of its origin and evolution (Eberhard, 1985; Eberhard et al., 1998; Simmons, 2014). Sexual selection is now widely acknowledged as being the major force driving the evolution of animal genitalia (Hosken \& Stockley, 2004; Simmons, 2014), but there is still little consensus on what are the dominant factors (such as male competition, male-female sexual conflict or pure female choice) or the main macroevolutionary trends generating their diversity (Simmons, 2014).

One of the few widely accepted general trends is the negative allometric scaling of most genital traits (Eberhard et al., 1998; Hosken et al., 2005; Eberhard, 2009), which suggests that genital morphology is often subject to stabilising selection. However, genitalia are also considered to be among the fastest evolving traits in arthropods, clearly differing in species which otherwise have very similar external morphologies (Eberhard, 1985; Rowe \& Arnqvist, 2011). This contrast - generalised stabilising selection but fast macroevolutionary diversification- raises the intriguing question of how divergence in genital characters is initiated and amplified to reach the extraordinary variability observed in many groups (Simmons et al., 2009; Hosken \& Stockley, 2004).

Most of the work on the evolution of genitalia has been conducted on reduced groups of species at limited temporal scales. There is very little quantitative data on the rate and mode of evolution of genital traits in diverse, old lineages, which could help to understand the origin of the large-scale variation in genital morphology (Hosken \& Stockley, 2004; Simmons, 2014). In this work we study the macroevolution of the size and complexity of male genitalia in a diverse insect lineage with a very uniform external morphology but extraordinary male genital variability, the aquatic beetle genus Limnebius (family Hydraenidae). Limnebius is divided in two sister lineages, one (subgenus Bilimneus) with uniformly small species $(0.8-1.1 \mathrm{~mm})$ with very simple genitalia, while the species of the other (subgenus Limnebius sensu stricto, "s.str." onwards) are much more variable in size $(0.9-2.5 \mathrm{~mm})$ and structure of the male genitalia (Fig. 1; Table 1; Perkins, 1980; Jäch, 1993; Rudoy et al., in press). We obtained different measures of the complexity and size of the male genitalia and the body size of males and females of a 
69 comprehensive representation of species of both subgenera to (i) determine the phylogenetic signal and possible correlated evolution of the studied traits; (ii) compare their evolutionary rates; and (iii) estimate the distribution of the reconstructed phenotypic change through the phylogeny. Our expectation is that the comparison of the rate and mode of evolution of the size and complexity of the male genitalia between two lineages with strikingly contrasting macroevolutionary trends will contribute to understand the origin of their extraordinary diversity.

\section{MATERIALS AND METHODS}

\section{Morphological measurements}

Limnebius includes ca. 150 species with an almost cosmopolitan distribution, all of them aquatic, living in all types of continental waters with the only exception of saline habitats (Perkins, 1980; Jäch, 1993; Hansen, 1998; Rudoy et al., in press). There is very few information on the ecology and behaviour of the species of Limnebius, including their sexual habits. In a recent work, Limnebius has been shown to be divided in two sister lineages with an estimated Oligocene origin, the subgenera Bilimneus and Limnebius s.str., with ca. 60 and 90 described species respectively (Rudoy et al., in press).

We characterised the size and complexity of the male genitalia of all species included in the molecular phylogeny (see below and Supplemental Information, Table S1). The structure of the female genitalia of Limnebius is poorly known, mostly due to the lack of sclerotized parts (Rudoy et al., in press), and was not studied here. We measured body length of adults (males, $l m$ and females, $l f$ ) as the sum of the individual maximum lengths of pronotum and elytra, as the different position of the articulation between the two could alter the total length when measured together. Similarly, the head was not measured, as in many specimens it was partly concealed below the pronotum. Measures were obtained with stereoscope microscopes equipped with an ocular micrometer.

Male genitalia (aedeagi) were dissected and mounted on transparent labels with dimethyl hydantoin formaldehyde (DMHF). For size measurements we used as a single value the average of each measure in all studied specimens of the same species (Supplemental Information, Table $\mathrm{S} 1)$. For the measures of complexity a single specimen was used, as species show in general a very constant shape of the aedeagus (Jäch, 1993; Rudoy et al., in press), and there is no evidence 
100 genitalia were always photographed in the same standard positions, orientated according to the

101 foramen in ventral and lateral views. We measured the maximum length of the male genitalia

102 ( $\lg$ ) on digital images obtained in the same standard position, orientated in ventral view as

103 determined by the foramen (Rudoy et al., in press). We did not include setae or apical

104 membranous structures but including appendages when they were longer than the median lobe

105 (as in e.g. some species of the L. nitidus group, Rudoy et al., in press). Measurements were

106 directly obtained from the digital images using ImageJ v.1.49 (National Institutes of Health, US,

107 http://imagej.nih.gov/ij/) (Supplemental Information, Fig. S1). We estimated experimental error

108 by measuring the same specimen of three species on three different sessions, using two sets of

109 images.

110 We used two different measures to characterise the complexity of the aedeagus:

111 (i) Perimeter (per) of the aedeagus in ventral view, including the median lobe and the main

112 appendages. We obtained an outline of the genitalia from digital images using ImageJ, with the

113 "free hand outline" option. The total perimeter was the sum of the values of the different parts of

114 the genitalia (median lobe and left parameter, plus main appendages if present, see Rudoy et al.

115 in press). We standardised the values by dividing the perimeter by the length of the aedeagus, to

116 obtain a measure of complexity by unit of length (Supplemental Information, Fig. S1, Table S1).

117 (ii) Fractal dimension $(f d)$. We estimated the fractal dimension of the outline of the

118 aedeagus in ventral view on images of standard size $(2100 \times 2100$ pixels, 2000 pixels from base to

119 apex of the aedeagus) with the software Fractal Dimension Estimator (http://www.fractal-

120 lab.org/index.html). This software estimates the Minkowski fractal dimension of bidimensional

121 images using the box-counting method (Falconer, 1990). The software converts the image to

122 binary data, selects the scaling window of the box, and counts how many boxes are necessary to

123 cover the image. The absolute value of the slope of a log-log graph of the scale with the number

124 of boxes is the fractal dimension of the image (Supplemental Information, Fig. S1, Table S1).

125

126 Phylogenetic analyses

127 We obtained a phylogeny of Limnebius using molecular data obtained from Rudoy et al. (in

128 press), which included 71 of the ca. 150 known species of the genus. The taxon sampling was

129 denser for the Palaearctic lineages in subgenus Limnebius s.str., including the full range of body

130 sizes and aedeagus structural variation (Rudoy et al., in press). Groups with the lowest number of 
131 sampled species show in general a more homogeneous and simpler aedeagus (L. mundus and $L$.

132 piceus groups and subgenus Bilimneus). We used as outgroup and to root the tree the genus

133 Laeliaena, considered to be sister to Limnebius based on multiple morphological

134 synapomorphies (Hansen, 1991; Jäch, 1995; Perkins, 1997; Beutel et al., 2003).

We reconstructed a phylogeny of the genus with Bayesian analyses in BEAST 1.8

136 (Drummond et al., 2012) using a combined data matrix with three partitions, (i) mitochondrial

137 protein coding genes (two coxl fragments plus $n a d 1$ ); (ii) mitochondrial ribosomal genes ( $r r n L$

138 plus $t r n L)$ and (iii) nuclear ribosomal genes ( $S S U$ plus $L S U$ ) (Supplemental Information, Table

$139 \mathrm{~S} 1)$. We used a Yule speciation process as the tree prior and an uncorrelated relaxed clock.

140 Analyses were run for 100 MY generations, ensuring that the number of generations after

141 convergence were sufficient as assessed with Tracer v1.6 (Drummond et al., 2012) and after

142 removal of a conservative $10 \%$ burn-in fraction.

143 To calibrate the phylogenetic tree we used the rates estimated in Cieslak et al. (2014) for

144 a related group (familiy Leiodidae, within the same superfamily Staphylinoidea, Beutel \&

145 Leschen, 2005) and the same gene combination based on the tectonic separation of the Sardinian

146 plate $33 \mathrm{Ma}$. We set as prior average mean rate a normal distribution with average 0.015

147 substitutions/site/Myr for the mitochondrial protein genes, 0.006 for the mitochondrial ribosomal

148 genes, and 0.004 for the nuclear ribosomal genes, all with a standard deviation of 0.001 . It must

149 be noted that for our objectives only relative rates are needed. An absolute calibration would

150 only be necessary to obtain absolute estimates of character change, which is not our main

151 objective and does not affect our conclusions.

152 We reconstructed the ancestral values of the morphological variables using the values of

153 the terminals (extant species) in BEAST 1.8. The reconstruction of ancestral characters should

154 ideally include a combination of extant and fossil data (e.g. Webster \& Purvis, 2002; Slater et al.,

155 2012; Bokma et al., 2015). However, as there are no known fossils of species of Limnebius we

156 can only use extant species. The use of extant species without fossil data has resulted in valuable

157 contributions to the study of character evolution (e.g. Cooper \& Purvis, 2010; Baker et al., 2015),

158 and in some cases it has been reported that the introduction of fossil data did not alter

159 substantially the conclusions obtained with extant species only (e.g. Puttick \& Thomas, 2015).

160 We implemented a Brownian movement model of evolution (BM), a null model of

161 homogeneous evolution in which variation accumulates proportionally with time, with 
162 incremental changes drawn from a random distribution with zero mean and finite constant

163 variance (Hunt \& Raboski, 2012; Adams, 2014). The reconstruction of ancestral values using a

164 BM model of evolution can be biased toward average or intermediate values (Pagel, 1999;

165 Finarelly \& Goswami, 2013), which together with the lack of fossil data can result in an

166 underestimation of the rates of evolution of some characters. Due to these limitations our

167 reconstruction needs to be understood as the simplest null model explaining the evolutionary

168 change in the studied characters. It must be noted though that some of our conclusions do not

169 depend on these reconstructions, and for others we compare two groups (Bilimneus and

170 Limnebius) likely affected by the same biases. In any case, all analyses were repeated using only

171 the data of the terminal branches, with a decreased power due to their lower number but less

172 prone to reconstruction biases. Terminal branches are truncated with respect to internal branches,

173 as they do not reflect a full interval between speciation events but the time elapsed since the last

174 cladogenetic split. This does not introduce any bias in our measures when change is anagenetic

175 (i.e. proportional to time), but will overestimate evolutionary rates if change occurs mostly at

176 speciation (i.e. according to punctuated models of evolution, Gingerich, 2009; Hunt \& Rabosky,

177 2014).

178

179 Statistical analyses

180 We studied the evolution of the morphological characters trough the full evolutionary path of

181 species (i.e. from root to tips) and in the individual branches, using phylogenetic ancestor-

182 descendant comparisons (PAD; Baker et al., 2015). We first deleted the terminals with species

183 with unknown males, as well as the duplicated specimen of L. cordobanus and the outgroup

184 (Laeliaena) (Supplemental Information, Table S1). We then estimated the phylogenetic signal of

185 the morphological variables in the whole tree using the $K$ metric proposed by Blomberg et al.

186 (2003), which tests whether the topology and branch lengths of a given tree better fits a set of tip

187 data compared with the fit obtained when the data have been randomly permuted. The higher the

$188 K$ statistic, the more phylogenetic signal in a trait. $K$ values of 1 correspond to a BM model,

189 which implies some degree of phylogenetic signal. $K$ values closer to zero correspond to a

190 random or convergent pattern of evolution, while $K$ values greater than 1 indicate strong

191 phylogenetic signal. We used the R package 'Picante' (Kembel et al., 2010) to compute $K$ and

192 the significance test. To test the possible decrease of power or the randomization test due to the 
193 low number of species in Bilimneus (15, vs. 50 in Limnebius s.str.) we randomly pruned the tree

194 of Limnebius s.str. to 15 species in 1,000 replicas and computed the $K$ values for all of them. We

195 also measured the correlation between the variables across the whole tree with a regression of

196 phylogenetic independent contrasts with the PDAP package in MESQUITE (Midfort et al.,

197 2011).

198 For the analyses of the individual branches of the tree (PADs) we compiled the

199 reconstructed values in BEAST of all variables for the initial and final node of each individual

200 branch, together with their length. We measured three values for each of the individual branches

201 (including terminals): (i) amount of phenotypic change, equal to the arithmetic difference

202 between the final and initial values of the branch; (ii) absolute amount of phenotypic change,

203 equal to the absolute value of the amount of phenotypic change; (iii) phenotypic change

204 measured in darwins (Haldane, 1949), computed as the absolute value of the natural logarithm of

205 the ratio between the final and initial values divided by the length of the branch in million years

206 (Myr) (Supplemental Information, Table S1). The use of the natural logarithm standardises the

207 change so it is proportional and directly comparable among species with different sizes (Haldane,

208 1949; Gingerich, 2009). Darwins are known to be strongly dependent on the length over which

209 are measured (Gingerich, 1983; Hunt, 2012; Hunt \& Rabosky, 2014), so to obtain the correlation

210 with time of the different variables we used instead the absolute amount of phenotypic change,

211 which showed a more linear relationship with branch length for most of the variables (see

212 Results). As individual branches are in principle independent from each other we analysed these

213 variables with standard statistical procedures (see e.g. Baker et al., 2015). We used a Bonferroni

214 correction for multiple tests and considered as significant a $p<0.01$ level (corresponding to five

215 morphological variables), although we also report some results at the standard $p<0.05$ level in

216 some of the analyses. In the comparisons between the two subgenera of Limnebius (Bilimneus

217 and Limnebius s.str.) we did not include the respective stem branches.

218

219 RESULTS

220 Phylogenetic signal and correlation between complexity and size

221 The reconstructed ancestral values of Bilimneus were in the upper range of the values of the 222 extant species, especially for measures of complexity ( $f d$ and $p e r)$. The reconstructed ancestral

223 values of the whole genus were, on the contrary, larger than any extant species of Bilimneus, 
224 with the only exception of the length of the genitalia $(l g)$ (Table 1). For the subgenus Limnebius 225 s.str. all reconstructed values, both of the genus and the subgenus, were within the range of the 226 extant species, and close to their centre of distribution (Table 1; Supplemental Information, 227 Tables S1, S2).

228 The phylogenetic signal $(K)$ was very significant for all variables both in the genus 229 Limnebius as a whole and the subgenus Limnebius s.str., with values close to 1 (i.e. a BM model) 230 for the measures of complexity ( $p e r$ and $f d$ ), clearly over 1 (i.e. with a stronger phylogenetic 231 signal) for the size of males and females ( $l f$ and $l m$ ), but with values lower than 1 (i.e. closer to a 232 global random distribution, although still significant) for the length of the genitalia ( $l g$ ) (Table 2). 233 In contrast, none of the variables had a significant $K$ in subgenus Bilimneus (Table 2). More than $23499 \%$ of the 1,000 pruned replicas of Limnebius s.str. maintained significant values of $K$ for all 235 variables at a $p<0.05$ level, and more than $90 \%$ at $p<0.01$, with the only exception of $l g$, 236 which become not significant (Table 2).

All pairwise correlations between the morphological variables were significant at a $p<$ 0.01 level as measured with PDAP both in Limnebius as a whole and in subgenus Limnebius s.str., with the exception of the correlation between $l g$ and the measures of complexity (per and fd) (Table 3). For Bilimneus, with a lower number of contrasts (14 vs 49 in Limnebius s.str.),

241 results were similar except that the length of the female (lf) was not significantly correlated with 242 any variable, and the length of the aedeagus $(l g)$ was significantly correlated with its fractal 243 dimension (Table 3).

\section{Rates of evolution}

There were no significant differences between terminal and all branches of the tree for any of the measured variables (including branch length) at a $p<0.01$ level, both in average value ( 2 tailed t-Student with unequal variances when differences were significant) or variance (Fisher's F-test) (Table 4). At a $p<0.05$ only for the measure of darwins the variances of per and $l f$ were larger in the terminals than in all branches, and the variance of $l g$ smaller (Table 4). Some of the largest increases or decreases of absolute change were in terminal branches (e.g. L. nitiduloides and $L$. cordobanus respectively, branches 94 and 72 in Supplemental Information, Fig. S2). 
255

256

257

258

259

260

261

262

263

264

265

266

267

268

269

270

271

272

273

274

275

276

277

278

279

280

281

282

283

284

285

Limnebius s.str. (Table 4). The lowest values of change were in $f d$, also in both subgenera. Males were in general more labile than females, with larger relative (but not absolute) differences in Bilimneus than in Limnebius s.str. (Table 4).

All comparisons of absolute change and darwins between Bilimneus and Limnebius s.str. were significantly different (always lower in Bilimneus) both for the variance and the average, with the only exception of the average $l g$, never significantly different between them regardless of how it was measured (Table 4; Supplemental Information, Fig. S3). When the sign of the change was considered variances were still significantly different, but only the average $f d$ showed differences (positive in Limnebius s.str. and negative in Bilimneus), although only at a $p$ $<0.05$ level (Table 4; Supplemental Information, Fig. S3).

Branches of Limnebius s.str. were on average significantly shorter and with a lower variance than that of Bilimneus (Table 4; Supplemental Information, Fig. S3). In the whole Limnebius and the subgenus Limnebius s.str. all the measures of absolute phenotypic change were significantly correlated with the length of the branch, with $l g$ having the lowest correlation (Fig. 2a; Table 5). On the contrary, for Bilimneus only $l g$ and per had significant correlations. When only the terminal branches were used the correlations between the measures of absolute phenotypic change and branch lengths were still significant at a $p<0.01$ level for Limnebius s.str. with the only exception of $l g$, but none of them was significant for Bilimneus (Fig. 2b; Table 5).

\section{Distribution of the reconstructed phenotypic change}

The amount of change in the individual branches for all variables was in general not significantly different from a normal distribution, as measured with a non-parametric Kolmogorov-Smirnov test. Only the variables of complexity when all the branches in the whole genus were considered could be said not to follow a normal distribution at a $p<0.01$ level (Table 6). When measured in darwins (and taking into account the sign of the branch), the amount of change of the individual variables was in general less normally distributed than when the change was measured with independence of the time, although at a $p<0.01$ level only per and $l f$ in the whole genus, and per in Limnebius s.str., were significantly non-normal (Table 6).

Despite the general normal distribution of the amount of change, in Bilimneus there was an excess of negative branches (i.e. a change to lower values) for all variables except for $\lg$. On 
286 the contrary, in Limnebius s.str. there were significantly more positive than negative changes for

287 the variables of complexity (Table 7). In both cases differences were mostly significant only at a

$288 p<0.05$ level, and in Limnebius s.str. only when all branches of the tree were considered. The

289 average amount of phenotypic change per branch was negative for all measures in Bilimneus and

290 positive in Limnebius s.str., but in all cases the 95\% confidence intervals included the zero (data

291 not shown).

292 For all measurements the general tendency was for a branch to have the same type of

293 change than that of the preceding. In Bilimneus this meant that the most frequent combination

294 was two branches with negative change, while in Limnebius s.str. two with positive change,

295 although $\lg$ had the lowest differences between the frequency of two consecutive positive or

296 negative branches in both subgenera (Table 7). The terminal branches showed the same tendency

297 except for the variables measuring length in Limnebius s.str., for which the most frequent

298 combination was two negative branches (Table 7).

299

300 DISCUSSION

301 We found clear differences in the macroevolution of the size and complexity of the aedeagus

302 between the two subgenera of Limnebius. In the subgenus with the highest diversity of body size

303 and aedeagus structure (Limnebius s.str.), both measures of complexity and the body size of

304 males and females had a strong phylogenetic signal. This signal was much lower for the size of

305 the genitalia, which evolved independently of complexity. Both measures of complexity had

306 values of the $K$ statistic closer to 1, suggesting a BM model of evolution (Blomberg et al., 2003).

307 This was supported by the strong correlation of both variables with the length of the branch (a

308 surrogate of time), as expected of characters evolving under a random walk (Gingerich, 2009;

309 Hunt, 2012; Hunt \& Raboski, 2014).

310 In the subgenus with the more homogeneous and simple genitalia, Bilimneus, the

311 tendency was for all measured variables to evolve more randomly, despite the significance of

312 some correlations. The correlation of the aedeagus size with its fractal dimension may be due

313 allometry resulting from its simplicity and the higher dimensionality of the variable $f d$. In

314 Limnebius s.str this allometric effect may be hidden by the larger variation in complexity. In

315 Bilimneus, length and perimeter of the genitalia were the only variables significantly correlated

316 with time, but in both cases the effect size was very low, and very similar in both subgenera for 
$317 \lg$ (Fig. 2). The amount of phenotypic change in Bilimneus for all variables was also smaller and

318 with a much smaller variance than in Limnebius s.str., except for $l g$. Despite the large difference

319 in size between some species between the two subgenera, there were no significant differences in

320 the average amount of change of the length of the genitalia in the individual branches, both with

321 the measure of absolute phenotypic change or in darwins.

322 There was some evidence for directional evolution in the complexity of the genitalia in

323 both subgenera, as revealed by the excess of branches with a positive change in Limnebius s.str.

324 and negative in Bilimneus, both individually and in combination to other branches of the same

325 sign. But this evidence for directional evolution was weak, and the fact that in all cases the 95\%

326 interval of the average of the measure of change included zero suggests that the dominant factor

327 was a random walk (Hunt, 2012). The directionality could also be partly due to the effect of the

328 hard boundary imposed by the intermediate condition of the reconstructed ancestor or the

329 artefacts introduced by the reconstruction method (Pagel, 1999; Hunt \& Raboski, 2014). These

330 biases for negative or positive change were, however, not present for the length of the genitalia,

331 which was more normally distributed and with a more balanced distribution of positive and

332 negative changes. Directional phenotypic change is rare in the fossil record, accounting for a

333 small fraction of the studied systems, likely due to the short time scale in which directional

334 selection needs to act to reach adaptive peaks (Hunt, 2007; Hunt \& Rabosky, 2014). The

335 evolution of most lineages seem to be driven by a complex interaction of random walk, stasis

336 and directional evolution, suggesting bouts of different types of evolutionary constraints in an

337 idiosyncratic sequence (Hunt, 2012; Hunt et al., 2015). Experimental results on the effect of

338 sexual selection agree with this idiosyncratic view of the macroevolution of the genitalia.

339 Selection can act differently on different genital traits, but there is no evidence of the existence

340 of directional trends acting through long evolutionary scales which may accelerate

341 macroevolutionary divergence (e.g. House \& Simmons, 2003; Simmons et al., 2009; Macagno et

342 al., 2011; Rowe \& Arnqvist, 2011; Simmons \& Garcia-Gonzalez, 2011;Hotzy et al., 2012;

343 Sakurai et al., 2012; Okuzaki \& Sota, 2014; Richmond, 2014; Dougherty et al., 2015).

344 Contrary to what happened with the evolution of complexity, the weaker phylogenetic

345 signal in the length of the male genitalia, weaker correlation with time and more normal

346 distribution of change would be compatible with an evolution dominated either by random

347 factors or by stasis (Hunt, 2012; Hunt \& Raboski, 2014). This would seem paradoxical, as 
348 genital length is the variable with the highest evolutionary rates in both subgenera, but it should

349 be considered that stasis is defined by the bias of the phenotypic variation of a trait, not by its

350 amplitude - that is, for the tendency of the trait to return to an overall optimal value (Hunt, 2012),

351 but not for the amplitude of variation around this optimum. It must also be considered that in our

352 PAD analyses we pooled branches from multiple independent lineages, which means that even

353 when the length of the genitalia may show a macroevolutionary pattern compatible with stasis

354 and stabilizing selection, it may still be subjected to directional selection in different lineages.

355 The main difference in the evolution of the male genitalia between the two subgenera

356 seems thus to be the strong contrast in the variance of the amount of phenotypic change. Whether

357 this difference was due to a constraint in the variation in Bilimneus or a relaxation in Limnebius

358 s.str. is at present unknown. The low variability of the size and complexity of the genitalia within

359 the sister genus of Limnebius, Laeliaena (Jäch, 1995; Rudoy et al., in press) suggest the

360 possibility that the higher variability of Limnebius s.str. is the derived condition, but the low

361 number of species of Laeliaena (three) precludes any firm conclusion. A potential key factor

362 could be the different relative size of males and females in the two subgenera of Limnebius:

363 while in Limnebius s.str. males are usually larger (sometimes much larger) than females, in

364 Bilimneus females are larger than males (Supplemental Information, Table S1), suggesting a

365 different role of sexual selection in the two subgenera.

366 There are two main potential biases affecting our conclusions, the lack of fossil data and

367 the use of a BM model in the reconstruction of ancestral values. As already noted, the lack of

368 fossil data in combination with a BM reconstruction model imply that all reconstructed values

369 (including the ancestors) should be within the range of the extant species, which may not be

370 accurate (Pagel, 1999). This may result in an underestimation of the variance and of the number

371 of shifts in the direction of change, i.e. a stronger tendency to linearity -but also a stronger

372 tendency to find that variables evolve under a BM model, which may led to some degree of

373 circularity. The analyses of the phylogenetic signal $(K)$ and the overall correlation between

374 variables were, however, not affected by these biases, but still clearly reflected difference

375 between subgenera and between the evolution of complexity and size of the aedeagus. Similarly,

376 the results of the PAD analyses using the terminal branches were in general in agreement with

377 that of all the branches of the tree, although with less statistical power due to their lower number. 


\section{CONCLUSIONS}

380 The extraordinary variation in body size and male genitalia in one of the lineages of the genus

381 Limnebius seems to be the result not of a clear directional trend, but of its larger

382 macroevolutionary variance. The complexity of the aedeagus in Limnebius s.str. largely evolved

383 in good agreement with a Brownian motion model, although we found evidence of weak

384 directional selection for respectively and increase or decrease of complexity in the two

385 subgenera, likely acting in some lineages and at limited temporal scales. In contrast, change in

386 the size of the genitalia, the variable with the highest rate of phenotypic evolution, was less

387 dependent on the phylogeny and time, and without any evidence of directional selection.

388

389

390

391

392

393

394

395

396

397

398

399

400

401

402

403

404

405

406

407

408

409

\section{ACKNOWLEDGEMENTS}

We are grateful to M.A. Jäch (NMW, Wien) and P. Perkins (MCZ, Harvard) for allowing us to study the collections of their institutions and for supporting this work in many ways, and A. Cieslak, A. Cordero (University of Vigo) and two anonymous Referees for comments to the manuscript. A.R. thanks M.A. Jäch for his help during his stay in the NMW.

\section{REFERENCES}

Adams DC. 2014. Quantifying and comparing phylogenetic evolutionary rates for shape and other high-dimensional phenotypic data. Systematic Biology 63:166-177 doi:10.1093/sysbio/syt105.

Baker J, Meade A, Pagel M, Venditti C. 2015. Adaptive evolution towards larger size in mammals. Proceeding of the National Academy of Sciences, USA 112:5093-5098 doi:10.1073/pnas.1419823112.

Beutel RG, Anton E, Jäch MA. 2003. On the evolution of adult head structures and the phylogeny of Hydraenidae (Coleoptera, Staphyliniformia). Journal of Zoological Systematics and Evolutionary Research 41:256-275.

Beutel RG, Leschen RAB (eds) 2005. Handbook of Zoology, Part 38: Volume 1: morphology and systematics (Archostemata, Adephaga, Myxophaga, Polyphaga partim). Berlin, Germany: Walter de Gruyter. 
410 Blomberg SP, Garland Jr T, Ives AR. 2003. Testing for phylogenetic signal in comparative

411

412

413

414

415

416

417

418

419

420

421

422

423

424

425

426

427

428

429

430

431

432

433

434

435

436

437

438

439

440 data: behavioral traits are more labile. Evolution 57:717-745.

Bokma F, Godinot M, Maridet O, Ladevèze S, Costeur L, Solé F, Gheerbrant E, Peigné S, Jacques F, Laurin AM. 2015. Testing for Depéret's Rule (body size increase) in mammals using combined extinct and extant data. Systematic Biology 65:98-108 doi:10.1093/sysbio/syv075.

Cieslak A, Fresneda J, Ribera I. 2014. Life history evolution and diversification in Leptodirini cave beetles. Proceedings of the Royal Society of London, Series B 281:20132978 doi:10.1098/rspb.2013.2978.

Cooper N, Purvis A. 2010 Body size evolution in mammals: complexity in tempo and mode. The American Naturalist 175:727-738 doi:10.1086/652466.

Dougherty LR, Rahman IA, Burdfield-Steel ER, Greenway EV, Shuker DM. 2015. Experimental reduction of intromittent organ length reduces male reproductive success in a bug. Proceedings of the Royal Society of London, Series B 282:20150724 doi:10.1098/rspb.2015.0724.

Drummond AJ, Suchard MA, Xie D, Rambaut A. 2012. Bayesian phylogenetics with BEAUti and the BEAST 1.7. Molecular Biology and Evolution 29:1969-1973 doi:10. 1093/molbev/mss075.

Dufour L. 1848. Anatomie générale des Dipteres. Annuare de Science Naturelle 1:244-264.

Eberhard WG. 1985. Sexual Selection and Animal Genitalia. Cambridge, Massachusetts, USA: Harvard University Press.

Eberhard WG. 2009. Static allometry and animal genitalia. Evolution 63:48-66 doi:10.1111/j.1558-5646.2008.00528.x.

Eberhard WG, Hüber BA, Rodriguez RL, Briceño RD, Salas I, Rodriguez V. 1998. One size fits all? Relationships between the size and degree of variation in genitalia and other body parts in twenty species of insects and spiders. Evolution 52:415-431.

Falconer K. 1990. Fractal geometry: mathematical foundations and applications. Chichester, UK: John Wiley.

Finarelli JA, Goswami A. 2013. Potential pitfalls of reconstructing deep time evolutionary history with only extant data, a case study using the Canidae (Mammalia, Carnivora). Evolution 67:3678-3685 doi:10.1111/evo.12222. 
441 Gingerich PD. 2009. Rates of evolution. Annual Review of Ecology, Evolution and Systematics

442

443

444

445

446

447

448

449

450

451

452

453

454

455

456

457

458

459

460

461

462

463

464

465

466

467

468

469

470

40: 657-675 doi:10.1146/annurev.ecolsys.39.110707.173457.

Haldane JBS. 1949. Suggestions as to the quantitative measurement of rates of evolution. Evolution 3:51-56.

Hansen M. 1991. A review of the genera of the beetle family Hydraenidae (Coleoptera). Steenstrupia 17:1-52.

Hansen M. 1998. World Catalogue of Insects. 1. Hydraenidae (Coleoptera). Stenstrup, DK: Apollo Books.

Hosken DJ, Minder AM, Ward PI. 2005. Male genital allometry in Scathophagidae (Diptera). Evolutionary Ecology 19:501-515 doi:10.1007/s10682-005-1023-z.

Hosken DJ, Stockley P. 2004. Sexual selection and genital evolution. Trends in Ecology and Evolution 19:87-93 doi:10.1016/j.tree.2003.11.012.

Hotzy C, Polak M, Ronn L, Arnqvist G. 2012. Phenotypic engineering unveils the function of genital morphology. Current Biology 22:2258-2261 doi:10.1016/j.cub.2012.10.009.

House CM, Simmons LW. 2003. Genital morphology and fertilization success in the dung beetle Onthophagus taurus: an example of sexually selected male genitalia. Proceedings of the Royal Society of London, Series B 270:447-455 doi:10.1098/rspb.2002.2266.

Hunt G. 2007. The relative importance of directional change, random walks, and stasis in the evolution of fossil lineages. Proceeding of the National Academy of Sciences, USA 104:18404-18408 doi:10.1073 pnas.0704088104.

Hunt G. 2012. Measuring rates of phenotypic evolution and the inseparability of tempo and mode. Paleobiology 38:351-373 doi:10.1666/11047.1.

Hunt G, Hopkins MJ, Lidgard S. 2015. Simplex versus complex models of trait evolution and stasis as a response to environmental change. Proceeding of the National Academy of Sciences, USA 112:4885-4890 doi:10.1073/pnas.1403662111.

Hunt G, Rabosky DL. 2014. Phenotypic evolution in fossil species: pattern and process. Annual Review of Earth and Planetary Sciences 42:421-441 10.1146/annurev-earth-040809152524.

Jäch MA. 1993. Taxonomic revision of the Palearctic species of the genus Limnebius Leach, 1815 (Coleoptera: Hydraenidae). Koleopterologische Rundschau 63:99-187. 
471 Jäch MA. 1995. Taxonomic synopsis of the genus Laeliaena Sahlberg, 1900 (Coleoptera:

472 Hydraenidae). Elytron 8(1994):35-41.

473 Kembel SW, Cowan PD, Helmus MR, Cornwell WK, Morlon H, Ackerly DD, Blomberg

474

475

476

477

478

479

480

481

482

483

484

485

486

487

488

489

490

491

492

493

494

495

496

497

498

499

500

501

SP, Webb CO. 2010. Picante: R tools for integrating phylogenies and ecology.

Bioinformatics 26:1463-1464 doi:10.1093/bioinformatics/btq166.

Macagno ALM, Pizzo A, Parzer HF, Palestrini C, Rolando A, Moczek AP. 2011. Shape - but not size - codivergence between male and female copulatory structures in Onthophagus beetles. PLoS ONE 6(12):e28893 doi:10.1371/journal.pone.0028893.

Midford PE, Garland Jr T, Maddison WP. 2011. PDAP Package of Mesquite. Version 1.16. http://mesquiteproject.org/pdap_mesquite/

Okuzaki Y, Sota T. 2014. How the length of genital parts affects copulation performance in a carabid beetle: implications for correlated genital evolution between the sexes. Journal of Evolutionary Biology 27:565-574 doi:10.1111/jeb.12323.

Pagel M. 1999. Inferring the historical patterns of biological evolution. Nature 401:877-884.

Perkins PD. 1980. Aquatic beetles of the family Hydraenidae in the Western Hemisphere: classification, biogeography and inferred phylogeny (Insecta: Coleoptera). Quaestiones Entomologicae 16:3-554.

Perkins PD. 1997. Life on the effective bubble: exocrine secretion delivery systems (ESDS) and the evolution and classification of beetles in the family Hydraenidae (Insecta: Coleoptera). Annals of the Carnegie Museum 66:89-207.

Puttick MN, Thomas GH. 2015. Fossils and living taxa agree on patterns of body mass evolution: a case study with Afrotheria. Proceedings of the Royal Society of London, Series B 282: 20152023 doi:10.1098/rspb.2015.2023.

Richmond P. 2014. The role of aedeagus size and shape in failed mating interactions among recently diverged taxa in the Drosophila mojavensis species cluster. BMC Evolutionary Biology 12:255.

Rowe L, Arnqvist G. 2011. Sexual selection and the evolution of genital shape and complexity in water striders. Evolution 66:40-54 doi:10.1111/j.1558-5646.2011.01411.x.

Rudoy A, Beutel RG, Ribera I. Evolution of the male genitalia in the genus Limnebius Leach, 1815 (Coleoptera, Hydraenidae). Zoological Journal of the Linnean Society doi: 10.1111/zoj.12402. 
502 Sakurai G, Himuro C, Kasuya E. 2012. Intra-specific variation in the morphology and the

503

504

505

506

507

508

509

510

511

512

513

514

515

516

517

518

519

520 benefit of large genital sclerites of males in the adzuki bean beetle (Callosobruchus chinensis). Journal of Evolutionary Biology 25:1291-1297 doi:10.1111/j.14209101.2012.02517.x.

Simmons LW. 2014. Sexual selection and genital evolution. Austral Entomology 53:1-17 doi:10.1111/aen.12053.

Simmons LW, García-González F. 2011. Experimental coevolution of male and female genital morphology. Nature Communications 2:374 doi:10.1038/ncomms1379.

Simmons LW, House CM, Hunt J, García-González F. 2009. Evolutionary response to sexual selection in male genital morphology. Current Biology 19:1442-1446 doi:10.1016/j.cub.2009.06.056.

Slater GJ, Harmon LJ, Alfaro ME. 2012. Integrating fossils with molecular phylogenies improves inference of trait evolution. Evolution 66:3931-3944 doi:10.1111/j.15585646.2012.01723.x.

Webster AJ, Purvis A. 2002. Testing the accuracy of methods for reconstructing ancestral states of continuous characters. Proceedings of the Royal Society of London, Series B 269:143149 doi:10.1098/rspb.2001.1873. 


\section{TABLES}

522

523 Table 1. Average values of the measured variables in the outgroup (Laeliaena), the

524 reconstructed ancestor of Limnebius, and the reconstructed ancestor and the extant species

525 (maximum and minimum values) of the two subgenera. per, perimeter; $f d$, fractal dimension; $l m$,

526 male body length; $l f$, female body length; $l g$, length of the male genitalia.

527

528

Laeliaena Limnebius Bilimneus Limnebius s.str.

\begin{tabular}{lcccccccc} 
& & ancestor & $\min$ & Max & ancestor & $\min$ & Max & ancestor \\
\hline per & 3.00 & 3.74 & 2.18 & 2.73 & 2.69 & 2.38 & 10.07 & 4.89 \\
fd & 1.28 & 1.24 & 1.16 & 1.20 & 1.19 & 1.18 & 1.42 & 1.28 \\
$l m$ & 1.42 & 1.25 & 0.82 & 1.07 & 0.99 & 0.92 & 2.45 & 1.44 \\
lf & 1.25 & 1.25 & 0.91 & 1.10 & 1.10 & 1.01 & 2.22 & 1.39 \\
$l g$ & 0.39 & 0.42 & 0.23 & 0.44 & 0.36 & 0.31 & 1.21 & 0.48
\end{tabular}

529 
530 Table 2. Values of the $K$ statistic for all measured variables in the genus Limnebius and its two 531 subgenera, including the randomized pruned 1000 replicas of the tree of Limnebius s.str. with 532 only 15 species (see Text). In bold, significant values at a $p<0.01$; with stars, significant values 533 at a $p<0.05$. See Table 1 for the codes of the measured variables.

534

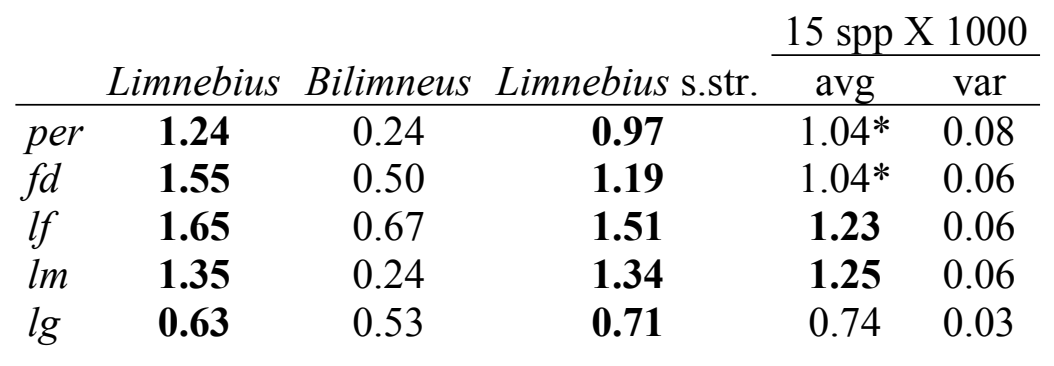

535

536 
537 Table 3. Correlation between the measured variables, as estimated with phylogenetic

538 independent contrasts. In bold, significant values at a $p<0.01$. See Table 1 for the codes of the 539 measured variables.

540

\begin{tabular}{|c|c|c|c|c|c|}
\hline & & per & $f d$ & If & $\operatorname{lm}$ \\
\hline Limnebius & $f d$ & 0.75 & & & \\
\hline \multirow[t]{3}{*}{$n=65$} & lf & 0.44 & 0.48 & & \\
\hline & $\operatorname{lm}$ & 0.53 & 0.50 & 0.87 & \\
\hline & $\lg$ & 0.11 & 0.31 & 0.53 & 0.53 \\
\hline Bilimneus & $f d$ & 0.66 & & & \\
\hline \multirow[t]{3}{*}{$\mathrm{n}=14$} & lf & -0.04 & 0.02 & & \\
\hline & $\operatorname{lm}$ & 0.75 & 0.73 & 0.38 & \\
\hline & $\lg$ & 0.41 & 0.70 & 0.08 & 0.71 \\
\hline Limnebius s.str. & $f d$ & 0.76 & & & \\
\hline \multirow[t]{3}{*}{$\mathrm{n}=49$} & lf & 0.43 & 0.47 & & \\
\hline & $\operatorname{lm}$ & 0.52 & 0.48 & 0.89 & \\
\hline & $\lg$ & 0.10 & 0.29 & 0.54 & 0.52 \\
\hline
\end{tabular}


543 Table 4. Average value and variance of the average of the measures of evolutionary change in

544 the individual branches of the phylogeny (PAD analyses, see Methods). In bold, significant

545 differences at a $p<0.01$, and with stars at a $p<0.05$, for the comparison between average (t-test)

546 or variance (F-test) between terminals and all branches of Limnebius, and between the subgenera

547 Bilimneus and Limnebius s.str. respectively. b.1., branch lengths. Measures in darwins x 10(-6).

548 See Table 1 for the codes of the measured variables, and Supplemental Information, Fig. S3 for a

549 graphic estimation of the density functions of the different comparisons.

550

\begin{tabular}{|c|c|c|c|c|c|c|c|}
\hline \multicolumn{2}{|c|}{ terminals } & \multicolumn{2}{|c|}{ all branches } & \multicolumn{2}{|c|}{ Bilimneus } & \multicolumn{2}{|c|}{ Limnebius s.str. } \\
\hline avg & var & avg & var & avg & var & avg & var \\
\hline 4.61 & 28.75 & 3.47 & 11.16 & 5.50 & 4.29 & 2.89 & 2.78 \\
\hline \multicolumn{8}{|c|}{ phenotypic change } \\
\hline-0.071 & 0.358 & 0.005 & 0.360 & -0.068 & 0.015 & 0.026 & 0.458 \\
\hline-0.002 & 0.000 & 0.000 & 0.000 & $-0.004 *$ & 0.000 & $0.001 *$ & 0.000 \\
\hline-0.007 & 0.004 & 0.000 & 0.006 & -0.009 & 0.001 & 0.003 & 0.008 \\
\hline-0.014 & 0.012 & 0.000 & 0.013 & -0.017 & 0.004 & 0.005 & 0.016 \\
\hline-0.009 & 0.004 & 0.000 & 0.003 & -0.003 & $0.002 *$ & 0.002 & $0.004^{*}$ \\
\hline \multicolumn{8}{|c|}{ absolute phenotypic change } \\
\hline r 0.423 & 0.182 & 0.373 & 0.220 & 0.102 & 9 & 0.450 & 0.253 \\
\hline 0.014 & 0.000 & 0.013 & 0.000 & 0.006 & 0.000 & 0.015 & 0.000 \\
\hline 0.045 & 0.002 & 0.048 & 0.004 & 0.022 & 0.000 & 0.055 & 0.004 \\
\hline 0.082 & 0.005 & 0.075 & 0.008 & 0.048 & 0.001 & 0.083 & 0.009 \\
\hline 0.047 & 0.002 & 0.040 & 0.002 & 0.033 & 0.001 & 0.043 & 0.002 \\
\hline \multicolumn{8}{|l|}{ darwins } \\
\hline er 0.048 & $0.005^{*}$ & 0.039 & $0.003 *$ & 0.011 & & 0.048 & 0.003 \\
\hline 0.005 & 0.000 & 0.005 & 0.000 & 0.002 & 0.000 & 0.006 & 0.000 \\
\hline 0.019 & $0.001 *$ & 0.017 & $0.001 *$ & 0.006 & 0.000 & 0.021 & 0.001 \\
\hline 0.030 & 0.001 & 0.023 & 0.001 & $0.014 *$ & 0.000 & $0.026^{*}$ & 0.001 \\
\hline 0.047 & 0.002 & 0.043 & 0.003 & 0.022 & 0.001 & 0.050 & 0.004 \\
\hline
\end{tabular}

551 
553 Table 5. Parameters of the linear regression between branch length and the measures of absolute

554 change for all and for terminal branches, and for the whole genus Limnebius, Bilimneus and

555 Limnebius s.str. In bold, significant values at a $p<0.01$ and confidence intervals including the

556 zero; with stars, significant values at a $p<0.05$. See Table 1 for the codes of the measured

557 variables and Fig. 2.

558

\begin{tabular}{lcr|rrr|rrr} 
& & & \multicolumn{3}{|c|}{$\mathbf{9 5 \%}$ intercept } & \multicolumn{3}{|c}{$\mathbf{9 5 \%}$ slope } \\
all branches & $\mathbf{R 2}$ & $\boldsymbol{p}$ & intercept & min & Max & slope & min & Max \\
\hline Limnebius & & & & & & & & \\
\hline per & $\mathbf{0 . 1 2 1}$ & $<\mathbf{0 . 0 0 0 1}$ & 0.203 & 0.091 & 0.316 & 0.049 & 0.026 & 0.072 \\
fd & $\mathbf{0 . 1 8 4}$ & $<\mathbf{0 . 0 0 0 1}$ & 0.006 & 0.003 & 0.010 & 0.002 & 0.001 & 0.003 \\
lf & $\mathbf{0 . 1 0 5}$ & $\mathbf{0 . 0 0 0}$ & 0.027 & 0.012 & 0.042 & 0.006 & 0.003 & 0.009 \\
lm & $\mathbf{0 . 1 5 8}$ & $<\mathbf{0 . 0 0 0 1}$ & 0.039 & 0.019 & 0.059 & 0.010 & 0.006 & 0.015 \\
lg & $\mathbf{0 . 0 8 3}$ & $\mathbf{0 . 0 0 1}$ & 0.028 & 0.017 & 0.038 & 0.004 & 0.002 & 0.006 \\
\hline Bilimneus & & & & & & & & \\
\hline per & $\mathbf{0 . 2 3 7}$ & $\mathbf{0 . 0 0 9}$ & 0.041 & $\mathbf{- 0 . 0 1 4}$ & $\mathbf{0 . 0 9 6}$ & 0.011 & 0.003 & 0.019 \\
fd & 0.133 & 0.057 & 0.003 & 0.000 & 0.007 & 0.001 & 0.000 & 0.001 \\
lf & 0.001 & 0.910 & 0.023 & 0.010 & 0.035 & 0.000 & $\mathbf{- 0 . 0 0 2}$ & $\mathbf{0 . 0 0 2}$ \\
lm & 0.138 & 0.052 & 0.030 & 0.007 & 0.053 & 0.003 & $\mathbf{0 . 0 0 0}$ & $\mathbf{0 . 0 0 7}$ \\
lg & $\mathbf{0 . 4 4 3}$ & $\mathbf{0 . 0 0 0}$ & 0.009 & $\mathbf{- 0 . 0 0 5}$ & $\mathbf{0 . 0 2 3}$ & 0.004 & 0.002 & 0.006 \\
\hline Limnebius sstr & & & & & & & & \\
\hline per & $\mathbf{0 . 3 7 0}$ & $<\mathbf{0 . 0 0 0 1}$ & 0.132 & 0.015 & 0.248 & 0.110 & 0.081 & 0.139 \\
fd & $\mathbf{0 . 4 3 9}$ & $<\mathbf{0 . 0 0 0 1}$ & 0.004 & 0.000 & 0.007 & 0.004 & 0.003 & 0.005 \\
lf & $\mathbf{0 . 3 2 0}$ & $<\mathbf{0 . 0 0 0 1}$ & 0.016 & 0.000 & 0.032 & 0.014 & 0.010 & 0.018 \\
lm & $\mathbf{0 . 3 3 1}$ & $<\mathbf{0 . 0 0 0 1}$ & 0.026 & 0.003 & 0.048 & 0.020 & 0.014 & 0.025 \\
$l g$ & $\mathbf{0 . 0 7 8}$ & $\mathbf{0 . 0 0 5}$ & 0.029 & 0.016 & 0.042 & 0.005 & 0.001 & 0.008 \\
\hline tg & & & & & & & &
\end{tabular}

\begin{tabular}{|c|c|c|c|c|c|c|c|c|}
\hline \multicolumn{9}{|c|}{$\begin{array}{l}\text { terminal b } \\
\text { Limnebius }\end{array}$} \\
\hline per & 0.015 & 0.326 & 0.362 & 0.205 & 0.519 & 0.014 & -0.014 & 0.042 \\
\hline$f d$ & $0.083^{*}$ & $0.020 *$ & 0.010 & 0.005 & 0.014 & 0.001 & 0.000 & 0.002 \\
\hline lf & 0.024 & 0.216 & 0.037 & 0.019 & 0.055 & 0.002 & -0.001 & 0.005 \\
\hline$l m$ & 0.031 & 0.163 & 0.039 & 0.025 & 0.054 & 0.002 & -0.001 & 0.004 \\
\hline $\lg$ & 0.038 & 0.118 & 0.065 & 0.040 & 0.091 & 0.004 & -0.001 & 0.008 \\
\hline \multicolumn{9}{|c|}{ Bilimneus } \\
\hline per & 0.109 & 0.229 & 0.078 & -0.040 & 0.195 & 0.008 & -0.005 & 0.021 \\
\hline$f d$ & 0.236 & 0.067 & 0.002 & -0.005 & 0.009 & 0.001 & 0.000 & 0.001 \\
\hline$l f$ & 0.092 & 0.273 & 0.038 & 0.013 & 0.062 & -0.001 & -0.004 & 0.001 \\
\hline $\operatorname{lm}$ & 0.006 & 0.786 & 0.076 & 0.035 & 0.117 & -0.001 & -0.005 & 0.004 \\
\hline $\lg$ & 0.256 & 0.054 & 0.027 & -0.001 & 0.055 & 0.003 & 0.000 & 0.006 \\
\hline \multicolumn{9}{|c|}{ Limnebius sstr } \\
\hline per & 0.241 & 0.000 & 0.258 & 0.088 & 0.428 & 0.080 & 0.039 & 0.121 \\
\hline$f d$ & 0.379 & $<0.0001$ & 0.006 & 0.002 & 0.011 & 0.003 & 0.002 & 0.004 \\
\hline lf & 0.248 & 0.000 & 0.021 & 0.001 & 0.042 & 0.010 & 0.005 & 0.015 \\
\hline $\operatorname{lm}$ & 0.156 & 0.004 & 0.049 & 0.017 & 0.080 & 0.011 & 0.004 & 0.019 \\
\hline $\lg$ & 0.008 & 0.535 & 0.041 & 0.023 & 0.060 & 0.001 & -0.003 & 0.006 \\
\hline
\end{tabular}


560 Table 6. Kolmogorov-Smirnov test of fit to a normal distribution of the phenotypic change and

561 darwins (considering the sign of the change) for all and terminal branches, and for Bilimneus and

562 Limnebius s.str. In bold, significant values at $p<0.01$ (i.e. the null hypothesis of a normal

563 distribution is rejected); with stars, significant values at $p<0.05$.

564

terminal

\begin{tabular}{llcccc} 
& var & Limnebius & branches & Bilimneus & Limnebius sstr \\
\cline { 2 - 6 } phenotpypic & per & $\mathbf{0 . 0 0 6}$ & 0.20 & 0.70 & 0.06 \\
change & $f d$ & $\mathbf{0 . 0 0 2}$ & 0.19 & 0.18 & $0.01^{*}$ \\
& $l f$ & $0.02^{*}$ & 0.45 & 0.79 & 0.11 \\
\multirow{4}{*}{ darwins } & $l m$ & 0.06 & 0.69 & 0.75 & 0.06 \\
& lg & 0.05 & 0.69 & 0.75 & $0.05^{*}$ \\
& per & $\mathbf{0 . 0 0 4}$ & $0.03^{*}$ & 0.16 & $\mathbf{0 . 0 0 7}$ \\
& fd & $0.01^{*}$ & 0.06 & $0.04^{*}$ & 0.06 \\
& $l f$ & $\mathbf{0 . 0 0 1}$ & $0.01^{*}$ & 0.31 & $0.03^{*}$ \\
& $l m$ & $0.01^{*}$ & 0.09 & 0.17 & 0.09 \\
& $l g$ & $0.01^{*}$ & 0.14 & 0.68 & 0.13
\end{tabular}

566 
567 Table 7. Number of branches with positive or negative change (A), and sign of a branch and the 568 preceding one (B), for all and terminal branches, and the genus Limnebius and the two subgenera 569 (Bilimneus and Limnebius s.str.). In bold, values significantly different at $p<0.01$ from a 570 binomial distribution with probability 50\% (positive vs. negative for A; two branches of the 571 same sign vs. two of different sign for B); with stars, significant values at $p<0.05$. all, all 572 branches; T, terminal branches; p.b., sign of the previous branch.

573

574

\begin{tabular}{|c|c|c|c|c|c|c|c|c|c|c|c|c|}
\hline \multirow{2}{*}{\multicolumn{3}{|c|}{ sign of the branch }} & \multicolumn{2}{|l|}{ per } & \multicolumn{2}{|l|}{$f d$} & \multicolumn{2}{|c|}{ lf } & \multicolumn{2}{|l|}{$\operatorname{lm}$} & \multicolumn{2}{|l|}{$\lg$} \\
\hline & & & + & - & + & - & + & - & + & - & + & - \\
\hline \multirow[t]{2}{*}{ Limnebius } & all & & 64 & 64 & 66 & 62 & 62 & 66 & $58 *$ & $70 *$ & 61 & 67 \\
\hline & $\mathrm{T}$ & & 28 & 37 & 30 & 35 & 32 & 33 & $27 *$ & $38 *$ & 29 & 36 \\
\hline \multirow[t]{2}{*}{ Bilimneus } & all & & 8* & $18 *$ & 7 & 19 & 9* & $17 *$ & 8* & $18 *$ & 13 & 13 \\
\hline & $\mathrm{T}$ & & 5 & 10 & $4^{*}$ & $11^{*}$ & $7 *$ & $8 *$ & $4^{*}$ & $11 *$ & 8 & 7 \\
\hline \multirow[t]{2}{*}{ Limnebius sstr } & all & & $54 *$ & $42 *$ & $56^{*}$ & $40 *$ & 51 & 45 & 48 & 48 & 45 & 51 \\
\hline & $\mathrm{T}$ & & 23 & 27 & 26 & 24 & 25 & 25 & 23 & 27 & 21 & 29 \\
\hline \multicolumn{2}{|c|}{$\begin{array}{l}\text { sign of the branch } \\
\text { vs preceding }\end{array}$} & p.b. & & & & & & & & & & \\
\hline \multirow[t]{4}{*}{ Limnebius } & all & + & 45 & 19 & 46 & 20 & 41 & 21 & 39 & 19 & 41 & 20 \\
\hline & & - & 29 & 35 & 26 & 36 & 21 & 45 & 23 & 47 & 25 & 42 \\
\hline & $\mathrm{T}$ & + & $20 *$ & 8* & $20 *$ & $10 *$ & 19 & 13 & 18 & 9 & 15 & 14 \\
\hline & & - & $17^{*}$ & $20 *$ & $17^{*}$ & $18^{*}$ & 10 & 23 & 14 & 24 & 15 & 21 \\
\hline \multirow[t]{4}{*}{ Bilimneus } & all & + & $3 *$ & $5 *$ & $2 *$ & $5 *$ & 3 & 6 & $4^{*}$ & $4 *$ & $7 *$ & $6^{*}$ \\
\hline & & - & $3 *$ & $15^{*}$ & $4^{*}$ & $15^{*}$ & 1 & 16 & $4^{*}$ & $14 *$ & $3 *$ & $10^{*}$ \\
\hline & $\mathrm{T}$ & + & $3 *$ & $2 *$ & 1 & 3 & 3 & 4 & 3 & 1 & 4 & 4 \\
\hline & & - & $1 *$ & 9* & 3 & 8 & 1 & 7 & 4 & 7 & 2 & 5 \\
\hline \multirow[t]{4}{*}{ Limnebius sstr } & all & + & $40^{*}$ & $14^{*}$ & 42 & 14 & 36 & 15 & 34 & 14 & 31 & 14 \\
\hline & & - & $24^{*}$ & $18^{*}$ & 22 & 18 & 18 & 27 & 18 & 30 & 21 & 30 \\
\hline & $\mathrm{T}$ & + & 17 & 6 & 19 & 7 & $16^{*}$ & $9 *$ & $15^{*}$ & $8 *$ & $11 *$ & $10^{*}$ \\
\hline & & - & 16 & 11 & 14 & 10 & $9 *$ & $16^{*}$ & $10^{*}$ & $17 *$ & $13 *$ & $16^{*}$ \\
\hline
\end{tabular}




\section{FIGURE LEGENDS}

577 Figure 1. Reconstructed phylogeny of the genus Limnebius, with estimated divergence times 578 (Ma). Dashed lines, terminal branches of species with no quantitative data for the aedeagus, 579 deleted in the analyses (see text). Numbers in nodes, posterior probability as obtained in BEAST. 580 Habitus photographs reflect maximum size variation within each subgenera; upper row: $L$.

581 cordobanus and L. fretalis, lower row: L. extraneus and L. oblongus (marked in bold in the tree). 582 Photos of the aedeagus in the right column standardised to the same size; scales refer to the size 583 of the aedeagus of $L$. fretalis (the largest, $1.21 \mathrm{~mm}$ ).

585 Figure 2. Bivariate plot of the branch length with the measure of phenotypic change for A) all 586 branches in the tree, and B) terminal branches only. Solid circles, species of Limnebius s.str.; 587 open circles, species of Bilimneus. See Table 5 for the parameters of the linear regressions. 
589

590

591

592

593

594

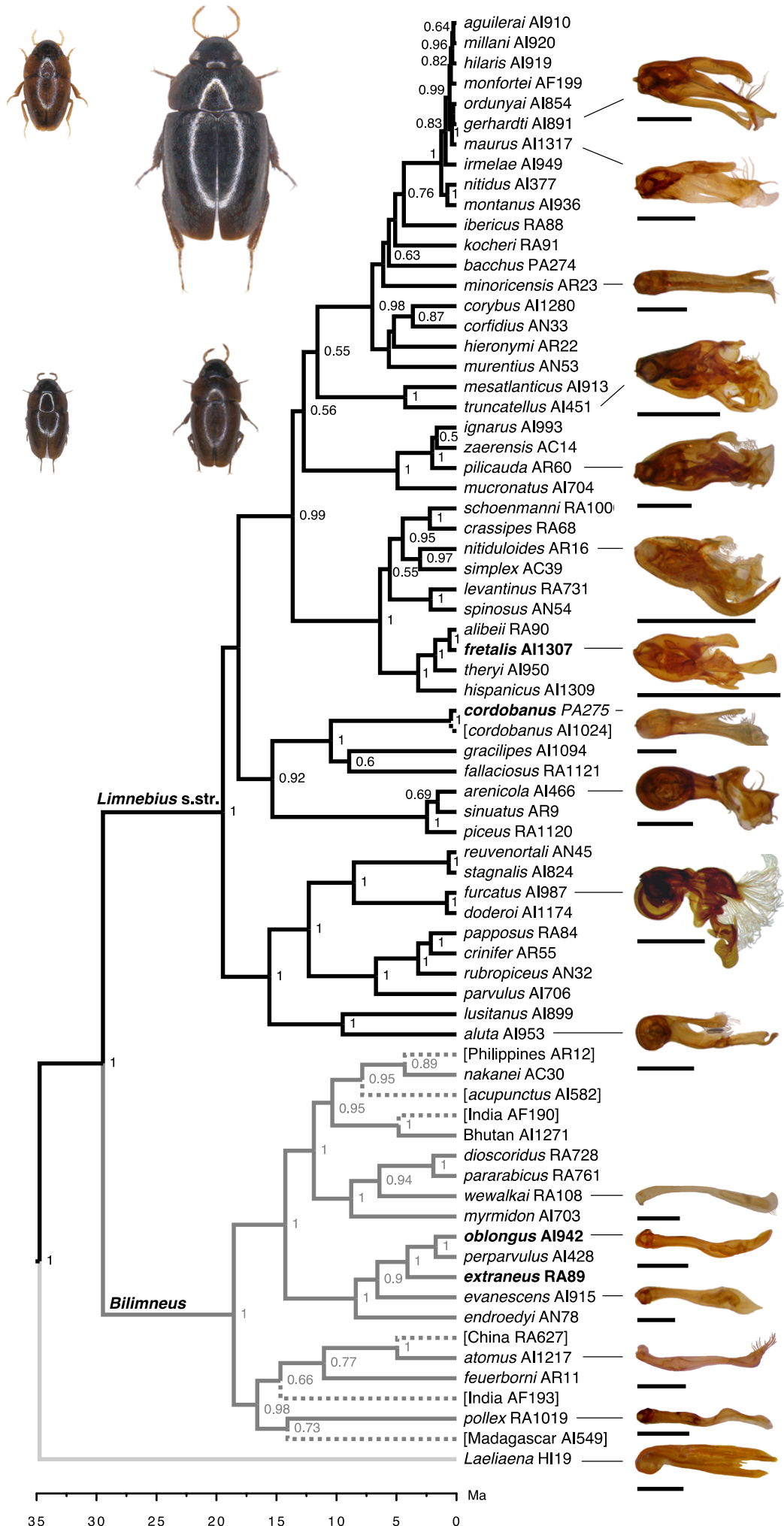


A
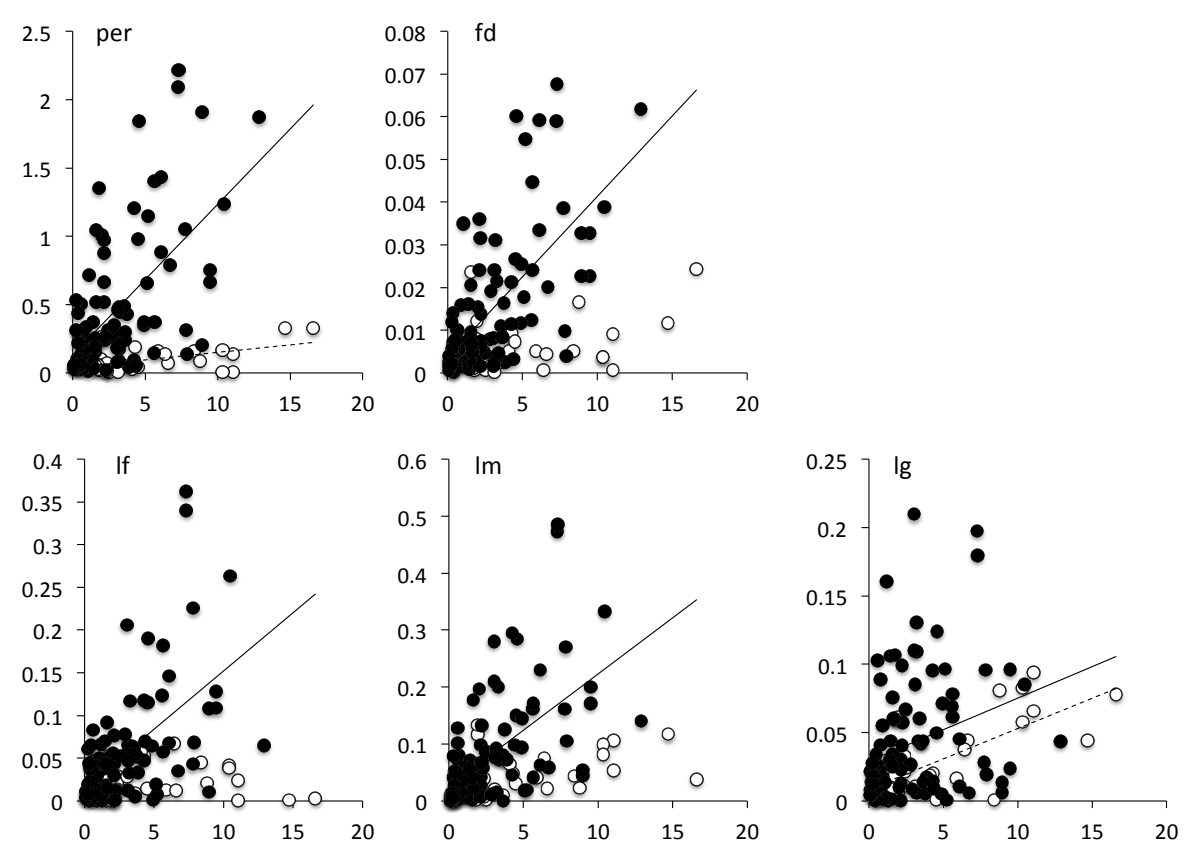

B
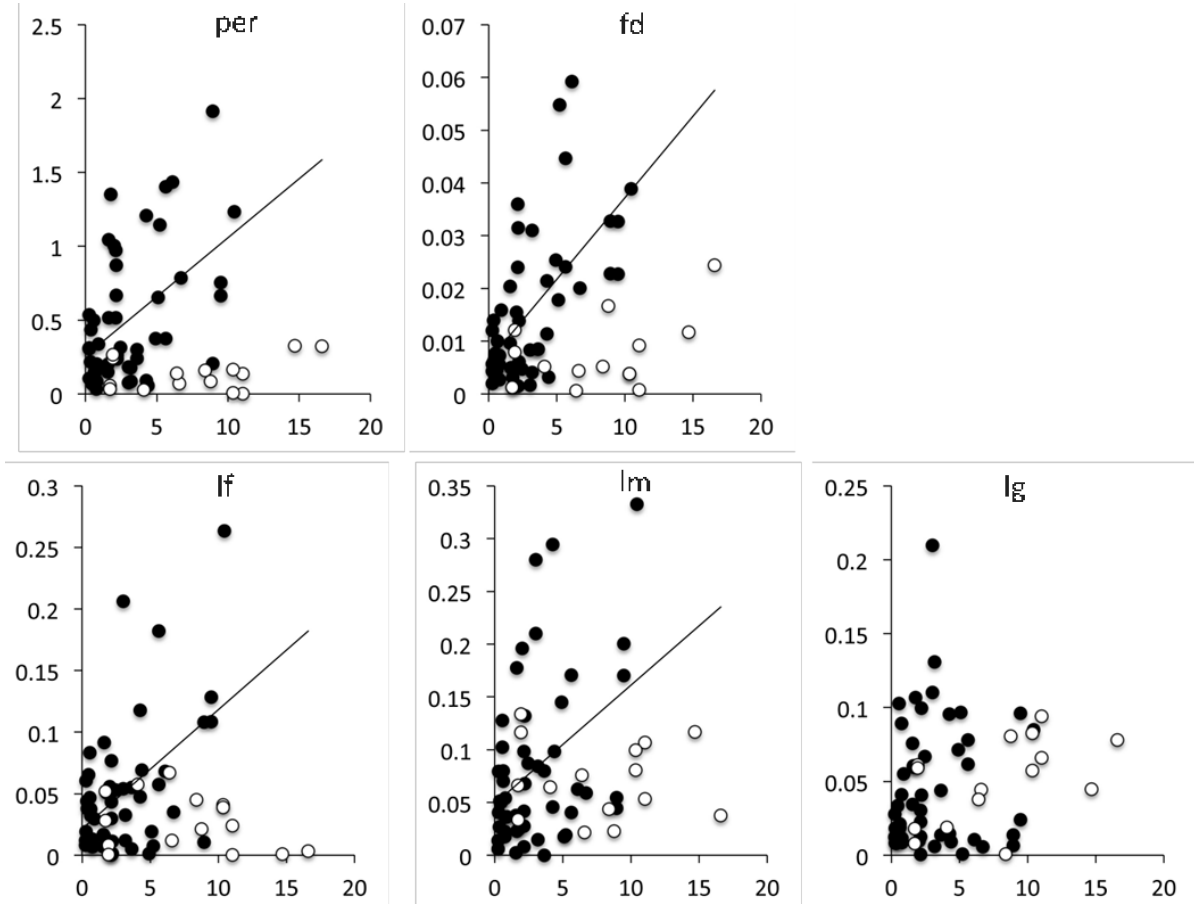
